\title{
Liberation from mechanical ventilator
}

\section{Objectives}

Discuss the variables that are used to indicate readiness to wean from mechanical ventilation Discuss the use of protocols to wean patients from ventilatory support Discuss the criteria used to indicate readiness for extubation Describe the most common reasons why patients fail to wean from mechanical ventilation.

\section{Predicted success rate}

$75 \%$ of mechanically ventilated patients are easy to be weaned off the ventilator with simple process $10-15 \%$ of patients require a use of a weaning protocol over a $24-72$ hours $5-10 \%$ require a gradual weaning over longer time $1 \%$ of patients become chronically dependent on MV

\section{Assessment criteria for weaning}

i. Neurological: (No sedation, GCS $>8$,Pain controlled)

ii. Respiratory: (NoWOB, PS <10,PEEP 5,FIO2-40\%,sat $>90 \%$, PF $>200$ )

iii. Cardiovascular : (Stable Hemodynamics, $\mathrm{Hb}>7$, Normal ECG)

\section{Ventilation status}

i. Intact ventilatory drive: ability to control their own level of ventilation

ii. Respiratory rate $<30$

iii. Minute ventilation of $<10 \mathrm{~L}$ to maintain $\mathrm{PaCO} 2$ in normal range

iv. $\mathrm{VD} / \mathrm{VT}<60 \%(\mathrm{Vd} / \mathrm{Vt}=0.320+0.0106(\mathrm{PaCO} 2$ - end-tidal carbon dioxide measurement) +0.003 (RR per minute) +0.0015 (age in years )

v. Functional respiratory muscles

\section{Intact airway protective mechanism}

i. Appropriate level of consciousness

ii. Cooperation

iii. Intact cough reflex

iv. Intact gag reflex

v. Functional respiratory muscles with ability to support a strong and effective cough

\section{Approaches to weaning}

i. Spontaneous breathing trials

ii. Pressure support ventilation (PSV) SIMV

iii. New weaning modes

\section{Maximal inspiratory pressure}

i. Negative Expiratory pressure must be more than $-20 \mathrm{cmH} 2 \mathrm{o}$

ii. Assures ability to mobilize secretions
Volume 2 Issue 4 - 2015

Patricia Colgan, Ibrahim Mohd Fawzy M

A Hassan, Mohammad Faisal Abdullah

Malmstrom, Abdul Aziz Ahmed M.Alhashemi, Thiruppathi Chockalingam, Ashok Parchani, MP Sujith, Mahesh Chandra, Damodaran CU, Flordevic P Guerra, Talib Yaseen

Hamad Medical Corporation, Qatar

Correspondence: Thiruppathi Chockalingam,Acting Assistan Director of Respiratory Therapy, Hamad Medical Corporation, 3050 Doha, Qatar, Tel (+974) 5531902I, (+974) 44392376, Fax (+974) 44391829, Email tchockalingam@hamad.qa

Received: March 12, 2015 | Published: April 10, 2015

\section{Shallow breathing index}

Index of rapid and shallow breathing $=\mathrm{RR} / \mathrm{Vt}$ in litre

\section{Single study results}

i. $\mathrm{RR} / \mathrm{Vt}>10595 \%$ wean attempts unsuccessful

ii. $\mathrm{RR} / \mathrm{Vt}<10580 \%$ successful

iii. One of the most predictive bedside parameters

\section{Spontaneous Breathing Trial (SBT)}
i. Explore Exclusion Criteria
ii. Assess Readiness for SBT
iii. If passed proceed sedation vacation for $30 \mathrm{~min}$
iv. Initiate SBT for 30 minutes
v. Assess the tolerance to SBT
vi. If SBT passed assess Readiness for Extubation

\section{Exclusion Criteria for SBT}
i. GCS $<8$ unsedated use of Neuro muscular drugs
ii. Neuro Muscular Disease with VC $<20 \mathrm{ml} / \mathrm{kg}$ or NIP $<20$
iii. RASS sedation and agitation scale +1 and higher, -3 and lower
iv. Immediate pending Invasive procedure
v. ICP $>20$ or needing RX in last 12 hours
vi. Ongoing Cardiac Ischemia
vii. Uncontrolled seizures 


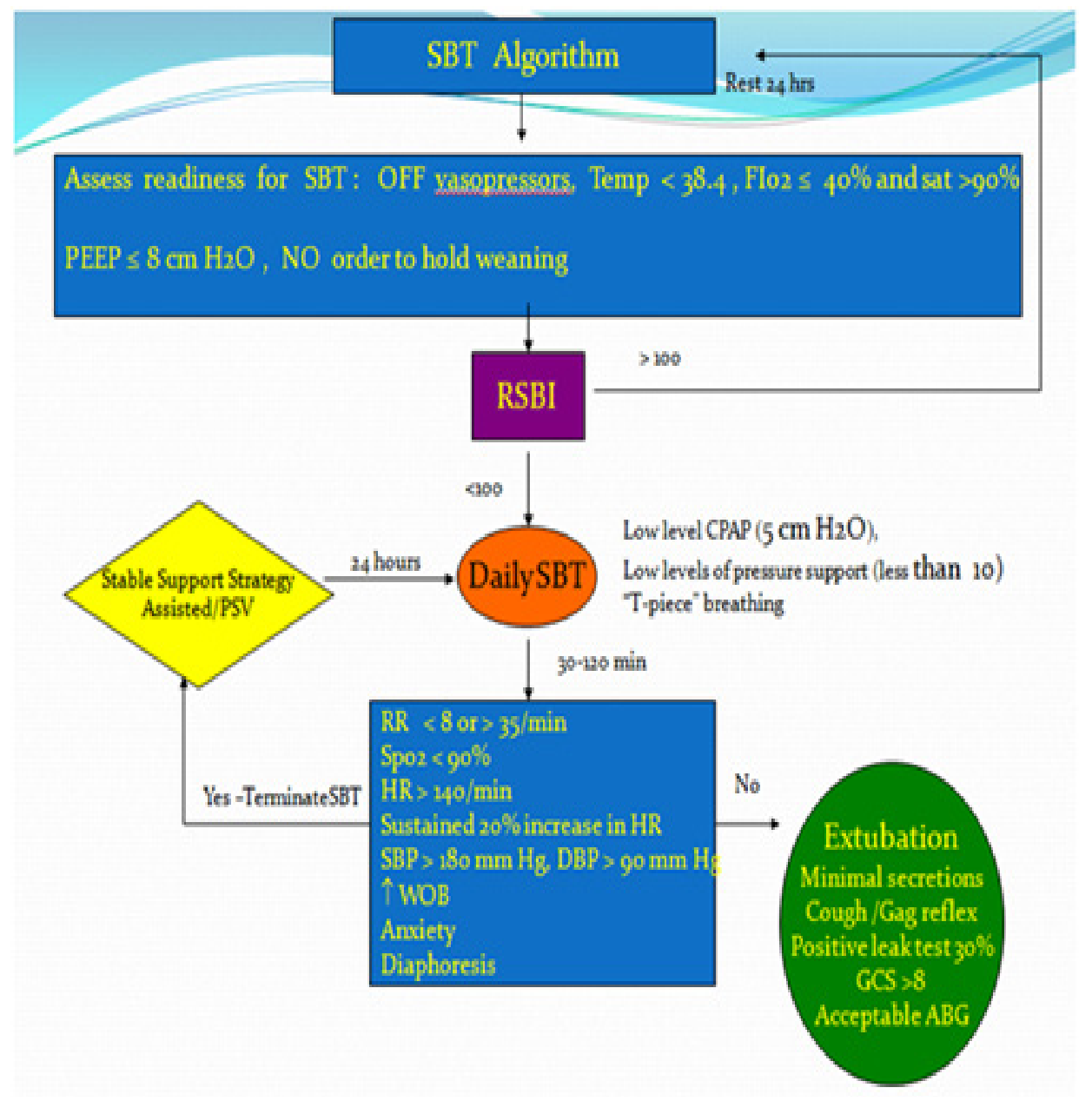

\section{Failure to wean}

i. Weaning to exhaustion

ii. Auto-PEEP

iii. Excessive work of breathing

iv. Poor nutritional status

v. Overfeeding

vi. Left heart failure

vii. Infection/fever

viii. Major organ failure

ix. Technical limitation

Appendix

\section{Protocols}

i. Developed by multidisciplinary team

ii. Implemented by respiratory therapists and nurses to make clinical decisions

iii. Results in shorter weaning times and shorter length of mechanical ventilation than physician-directed weaning

\section{Points to remember}

i. The primary prerequisite for weaning is reversal of the indication of mechanical ventilation

ii. Adequate gas exchange should be present with minimal oxygenation and ventilatory support before weaning is attempted

iii. The function of all organ systems should be optimized, electrolytes should be normal, and nutrition should be adequate before weaning is attempted

iv. The most successful predictor of weaning is RSBI $<100$

v. Maximum inspiratory pressure is the best predictor of weaning failure

vi. Ventilatory discontinuation should be done if patient tolerates SBT for 30-120minutes

vii. Use of liberation and weaning protocol facilitates the process and decreases the ventilator length of stay. ${ }^{1-4}$

\section{Acknowledgements}

None.

\section{Conflict of interest}

The author declares no conflict of interest. 


\section{References}

1. J bras. pneumol. São Paulo Sept./Oct. 2011;37(5).

2. http://dx.doi.org/10.1590/S1806-37132011000500016
3. Journal Brasileirode Pneumologia

4. AARC 